\title{
A special evolution equation used in the analysis of the stochastic Navier-stokes equation
}

\author{
Hannelore LISEI \\ Technische Universität Berlin \\ Berlin, Germany
}

Received for ROSE March 6, 2000

Abstract-We investigate a stochastic evolution equation of a special type. We use it to develop a linear approximation method for the solution of an equation of Navier-Stokes type.

\section{INTRODUCTION}

The stochastic Navier-Stokes equation has important physical and technical applications. It describes the behavior of a viscous velocity field of an incompressible liquid influenced by random internal and external perturbations. Several approximation methods were developed for this equation; for example, the Galerkin method was investigated by A. Bensoussan [1], H. Breckner [3], M. Capinski, D. Gatarek [4], A. I. Komech, M. I. Vishik [9], B. Schmalfuß [14], M. Viot [17]. Other approximation methods involve the approximation of the Wiener process by smooth processes (see W. Grecksch, B. SchmalfuB [7]), time discretizations (F. Flandoli, V. M. Tortorelli [6]), approximations of Wong-Zakai type (K. Twardowska [16]), the diffusion approximation technique (J. F. Clouet [5]).

The Galerkin method is useful to prove the existence of the solution, but it is complicated for numerical developments because it involves nonlinear terms. In our paper we give a new approximation method by making use of linear evolution equations (see equations $\left(\hat{P}_{n}\right)$ in Section 5), which are easier to study. We also prove that the approximations converge in mean square to the solution of the stochastic Navier-Stokes equation. This approximation method make use of stochastic evolution equations of the special type

$$
\begin{aligned}
\left(P_{\Psi, \Gamma}\right) \quad \mathbf{Z}_{\Psi, \Gamma}(t)+\int_{0}^{t} \mathcal{A} \mathbf{Z}_{\Psi, \Gamma}(s) \mathrm{d} s & =a_{0}+\int_{0}^{t} \mathcal{B}\left(X(s), \mathbf{Z}_{\Psi, \Gamma}(s)\right)+\mathcal{B}\left(\mathbf{Z}_{\Psi, \Gamma}(s), Y(s)\right) \mathrm{d} s \\
& +\int_{0}^{t} \Psi(s) \mathrm{d} s+\int_{0}^{t} \mathcal{G}\left(s, \mathbf{Z}_{\Psi, \Gamma}(s)\right) \mathrm{d} w(s)+\int_{0}^{t} \Gamma(s) \mathrm{d} w(s),
\end{aligned}
$$

assuming that the stochastic processes are defined on a given complete probability space, the Wiener process $w$ is given in advance and the hypothesis on $\mathcal{A}, \mathcal{B}, X, Y, \mathcal{G}, \Gamma, \Psi$ mentioned in Section 3 are fulfilled. Bereitgestellt von | Technische Universität Berlin 
In our paper we prove the existence of the solution of this type of equations (see Theorem 4) and this is the basic result for the development of the approximation method presented in Section 5. We also give a weak convergence property (see Lemma 4.3) for the solutions of equations of the type $\left(P_{\Psi, \Gamma}\right)$. This result is used to prove the existence of optimal controls, as soon as in the formulation of a stochastic minimum principle for the control problem of the stochastic Navier-Stokes equation (see [2], [11]).

Further applications of the equations $\left(P_{\Psi, \Gamma}\right)$ may be formulated; those equations are closely related to stochastic equations of Navier-Stokes type and are useful in their investigation.

The development and implementation of numerical methods for this type of equations remains an open problem for further research. For numerical solutions of stochastic differential equations we refer the reader to the book of P. Kloeden and E. Platen [8].

\section{NOTATION}

$\rightarrow$

$(\Omega, \mathcal{F}, \mathbf{P})$

$\left(\mathcal{F}_{t}\right)_{t \in[0, T]}$

$V^{*}$

$\left\langle v^{*}, v\right\rangle$

$\mathrm{J}$

$\mathcal{L}_{V}^{2}(\Omega \times[0, T])$

weak convergence (in the sense of functional analysis)

complete probability space

right continuous filtration such that $\mathcal{F}_{0}$ contains all $\mathcal{F}$-null sets dual space of the reflexive Banach space $V$

the application of $v^{*} \in V^{*}$ on $v \in V$

duality map $\mathrm{J}: V \rightarrow V^{*}$

space of all $\mathcal{F} \times B([0, T])$-measurable processes $u: \Omega \times[0, T] \rightarrow V$

that are adapted to the filtration $\left(\mathcal{F}_{t}\right)_{t \in[0, T]}$ and

$\mathbf{E} \int_{0}^{T}\|u(t)\|_{V}^{2} \mathrm{~d} t<\infty$

$\mathcal{L}_{V}^{\infty}(\Omega \times[0, T]) \quad$ space of all $\mathcal{F} \times B([0, T])$-measurable processes $u: \Omega \times[0, T] \rightarrow V$ that are adapted to the filtration $\left(\mathcal{F}_{t}\right)_{t \in[0, T]}$ and for a.e. $(\omega, t)$ bounded

$\mathcal{D}_{V}(\Omega \times[0, T]) \quad$ set of $\xi \in \mathcal{L}_{V}^{\infty}(\Omega \times[0, T])$ with $\xi=v \phi, v \in V, \phi \in \mathcal{L}_{\mathbb{R}}^{\infty}(\Omega \times[0, T])$

$\mathcal{D}_{V}(\Omega)$ set of $\xi \in \mathcal{L}_{V}^{\infty}(\Omega)$ with $\xi=v \phi, v \in V, \phi \in \mathcal{L}_{\mathbb{R}}^{\infty}(\Omega)$

$\Delta_{Q}(t)$ notation for $\exp \left\{-\frac{b}{\nu} \int_{0}^{t}\|Q(s)\|_{V}^{2} \mathrm{~d} s\right\}$, where $(Q(t))_{t \in[0, T]}$ is a $V$-valued stochastic process; $b, \nu$ are positive constants

\section{ASSUMPTIONS}

First we state the assumptions about the stochastic evolution equation that will be considered.

(i) $(\Omega, \mathcal{F}, P)$ is a complete probability space and $\left(\mathcal{F}_{t}\right)_{t \in[0, T]}$ is a right continuous filtration such that $\mathcal{F}_{0}$ contains all $\mathcal{F}$-null sets. $(w(t))_{t \in[0, T]}$ is a real valued standard $\mathcal{F}_{t}$-Wiener process.

(ii) $\left(V, H, V^{*}\right)$ is an evolution triple (see [20], p. 416), where $\left(V,\|\cdot\|_{V}\right)$ and $(H,\|\cdot\|)$ are separable Hilbert spaces, and the embedding operator $V \hookrightarrow H$ is assumed to be compact. We denote by $(\cdot$, . Gethe scalar productinche 
(iii) $\mathcal{A}: V \rightarrow V^{*}$ is a linear operator such that $\langle\mathcal{A} v, v\rangle \geq \nu\|v\|_{V}^{2}$ for all $v \in V$ and $\langle\mathcal{A} u, v\rangle=\langle\mathcal{A} v, u\rangle$ for all $u, v \in V$, where $\nu>0$ is a constant and $\langle\cdot, \cdot\rangle$ denotes the dual pairing.

(iv) $\mathcal{B}: V \times V \rightarrow V^{*}$ is a bilinear operator such that

$$
\langle\mathcal{B}(u, v), v\rangle=0 \quad \text { for all } u, v \in V
$$

and there exists a positive constant $b>0$ such that

$$
|\langle\mathcal{B}(u, v), z\rangle|^{2} \leq b\|z\|_{V}^{2}\|u\|\|u\|_{V}\|v\|\|v\|_{V} \quad \text { for all } u, v, z \in V .
$$

(v) $X, Y \in \mathcal{L}_{V}^{2}(\Omega \times[0, T])$ are arbitrary processes with almost surely continuous trajectories in $H$ and

$$
\mathbf{E} \sup _{t \in[0, T]}\|X(t)\|^{2}<\infty, \quad \mathbf{E} \sup _{t \in[0, T]}\|Y(t)\|^{2}<\infty .
$$

(vi) $\Psi \in \mathcal{L}_{V \cdot}^{2}(\Omega \times[0, T]), \Gamma \in \mathcal{L}_{H}^{2}(\Omega \times[0, T])$.

(vii) $\mathcal{G}:[0, T] \times H \rightarrow H$ is a mapping satisfying

(a) $\|\mathcal{G}(t, u)-\mathcal{G}(t, v)\|^{2} \leq \lambda\|u-v\|^{2}$ for all $t \in[0, T], u, v \in H$, where $\lambda$ is a positive constant;

(b) for each $t \in[0, T]$ the mapping $\mathcal{G}(t, \cdot): H \rightarrow H$ is linear;

(c) $\mathcal{G}(\cdot, v) \in \mathcal{L}_{H}^{2}[0, T]$ for all $v \in H$.

(viii) $a_{0}$ is a $H$-valued $\mathcal{F}_{0}$-measurable random variable such that $\mathbf{E}\left\|a_{0}\right\|^{4}<\infty$.

\section{A SPECIAL STOCHASTIC EVOLUTION EQUATION}

In this section we investigate the existence and some properties of the solution of the following stochastic evolution equation

$$
\begin{aligned}
\left(P_{\Psi, \Gamma}\right) \quad\left(Z_{\Psi, \Gamma}(t), v\right) & +\int_{0}^{t}\left\langle\mathcal{A} Z_{\Psi, \Gamma}(s), v\right\rangle \mathrm{d} s \\
& =\left(a_{0}, v\right)+\int_{0}^{t}\left\langle\mathcal{B}\left(X(s), Z_{\Psi, \Gamma}(s)\right)+\mathcal{B}\left(Z_{\Psi, \Gamma}(s), Y(s)\right), v\right\rangle \mathrm{d} s \\
& +\int_{0}^{t}\langle\Psi(s), v\rangle \mathrm{d} s+\int_{0}^{t}\left(\mathcal{G}\left(s, Z_{\Psi, \Gamma}(s)\right), v\right) \mathrm{d} w(s)+\int_{0}^{t}(\Gamma(s), v) \mathrm{d} w(s)
\end{aligned}
$$

for all $v \in V, t \in[0, T]$, and a.e. $\omega \in \Omega$, where the stochastic integral is understood in the Ito sense.

For an arbitrary natural number $M \in \mathbb{N}$ and a $V$-valued process $(Q(t))_{t \in[0, T]}$ with 
we introduce the following stopping times

$$
\begin{gathered}
\tilde{\mathcal{T}}_{M}^{Q}=\left\{\begin{array}{l}
T, \text { if } \sup _{t \in[0, T]}\|Q(t)\|^{2}<M \\
\inf \left\{t \in[0, T]:\|Q(t)\|^{2} \geq M\right\}, \quad \text { otherwise, }
\end{array}\right. \\
\tilde{\mathcal{T}}_{M}^{Q}=\left\{\begin{array}{l}
T, \text { if } \int_{0}^{T}\|Q(s)\|_{V}^{2} \mathrm{~d} s<M \\
\inf \left\{t \in[0, T]: \int_{0}^{t}\|Q(s)\|_{V}^{2} \mathrm{~d} s \geq M\right\}, \quad \text { otherwise. }
\end{array}\right.
\end{gathered}
$$

We define

$$
\mathcal{T}_{M}^{Q}:=\min \left\{\tilde{\mathcal{T}}_{M}^{Q}, \hat{\mathcal{T}}_{M}^{Q}\right\}
$$

Now we prove a property which is very useful in proving convergence results.

Proposition 4.1 Let $\left(\mathcal{T}_{M}\right)$ and $\mathcal{T}$ be stopping times, such that

$$
\lim _{M \rightarrow \infty} \mathbf{P}\left(\mathcal{T}_{M}<\mathcal{T}\right)=0
$$

Let $\left(Q_{n}\right)$ be a sequence of processes from the space $\mathcal{L}_{\mathbb{R}}^{2}([0, T] \times \Omega)$ such that for each fixed $M$ we have

$$
\lim _{n \rightarrow \infty} \mathbf{E}\left|Q_{n}\left(\mathcal{T}_{M}\right)\right|=0
$$

and there exists a positive constant $c$ independent of $n$ such that

$$
\mathbf{E}\left|Q_{n}(\mathcal{T})\right|^{2}<c \quad \text { for all } n \in \mathbb{N} \text {. }
$$

Then

$$
\lim _{n \rightarrow \infty} \mathbf{E}\left|Q_{n}(\mathcal{T})\right|=0
$$

Proof. Let $\varepsilon, \delta>0$. There exists $M_{0} \in \mathbb{N}$ such that

$$
\mathbf{P}\left(\mathcal{T}_{M_{0}}<\mathcal{T}\right) \leq \frac{\varepsilon}{2}
$$

By the hypothesis it follows that for this $M_{0}$ we have

$$
\lim _{n \rightarrow \infty} \mathbf{E}\left|Q_{n}\left(\mathcal{T}_{M_{0}}\right)\right|=0
$$

Consequently, there exists $n_{0} \in \mathbb{N}$ such that

$$
\frac{1}{\delta} \mathrm{E}\left|Q_{n}\left(\mathcal{T}_{M_{0}}\right)\right| \leq \frac{\varepsilon}{2}
$$

for all $n \geq n_{0}$. We write 


$$
\begin{aligned}
\mathbf{P}\left(\left|Q_{n}(\mathcal{T})\right| \geq \delta\right) & \leq \mathbf{P}\left(\mathcal{T}_{M_{0}}<\mathcal{T}\right)+\mathbf{P}\left(\left\{\mathcal{T}_{M_{0}}=\mathcal{T}\right\} \wedge\left\{\left|Q_{n}(\mathcal{T})\right| \geq \delta\right\}\right) \\
& \leq \frac{\varepsilon}{2}+\mathbf{P}\left(\left|Q_{n}\left(\mathcal{T}_{M_{0}}\right)\right| \geq \delta\right) \leq \frac{\varepsilon}{2}+\frac{1}{\delta} \mathbf{E}\left|Q_{n}\left(\mathcal{T}_{M_{0}}\right)\right|<\frac{\varepsilon}{2}+\frac{\varepsilon}{2}=\xi
\end{aligned}
$$

for all $n \geq n_{0}$. Hence for all $\delta>0$ we get

$$
\lim _{n \rightarrow \infty} \mathbf{P}\left(\left|Q_{n}(\mathcal{T})\right| \geq \delta\right)=0
$$

Therefore, the sequence $\left(\left|Q_{n}(\mathcal{T})\right|\right)$ converges in probability to zero. From the hypothesis it follows that this sequence is uniformly integrable (with respect to $\omega \in \Omega$ ). Hence it converges also in mean to zero

$$
\lim _{n \rightarrow \infty} \mathbf{E}\left|Q_{n}(\mathcal{T})\right|=0
$$

The proposition is proved.

For each $M \in \mathbb{N}$ let $\mathcal{T}_{M}:=\min \left\{\mathcal{T}_{M}^{X}, \mathcal{T}_{M}^{Y}\right\}$. From the properties of the stopping times it follows that

$$
\lim _{M \rightarrow \infty} \mathcal{T}_{M}=T \text { for a.e. } \omega \in \Omega
$$

as soon as

$$
\mathbf{P}\left(\bigcup_{M=1}^{\infty}\left\{\mathcal{T}_{M}=T\right\}\right)=1
$$

We define $X^{M}(t):=X\left(t \wedge \mathcal{T}_{M}\right), Y^{M}(t):=Y\left(t \wedge \mathcal{T}_{M}\right)$ for all $t \in[0, T]$.

For each $M \in \mathbb{N}$ we consider:

$$
\begin{aligned}
\left(P_{\Psi, \Gamma}^{M}\right) \quad & \left(Z_{\Psi, \Gamma}^{M}(t), v\right)+\int_{0}^{t}\left\langle\mathcal{A} Z_{\Psi, \Gamma}^{M}(s), v\right\rangle \mathrm{d} s \\
= & \left(a_{0}, v\right)+\int_{0}^{t}\left\langle\mathcal{B}\left(X^{M}(s), Z_{\Psi, \Gamma}^{M}(s)\right)+\mathcal{B}\left(Z_{\Psi, \Gamma}^{M}(s), Y^{M}(s)\right), v\right\rangle \mathrm{d} s \\
& +\int_{0}^{t}\langle\Psi(s), v\rangle \mathrm{d} s+\int_{0}^{t}\left(\mathcal{G}\left(s, Z_{\Psi, \Gamma}^{M}(s)\right), v\right) \mathrm{d} w(s)+\int_{0}^{t}(\Gamma(s), v) \mathrm{d} w(s)
\end{aligned}
$$

for all $v \in V, t \in[0, T]$, and a.e. $\omega \in \Omega$.

Let $h_{1}, h_{2}, \ldots, h_{n}, \ldots \in H$ be the eigenvectors of the operator $\mathcal{A}$, for which we consider the domain of definition $\operatorname{Dom}(\mathcal{A})=\{v \in V \mid \mathcal{A} v \in H\}$. These eigenvectors form an orthonormal base in $H$ and they are orthogonal in $V$ (see [10], p. 110).

For each $n \in \mathbb{N}$ we consider $H_{n}:=\operatorname{sp}\left\{h_{1}, h_{2}, \ldots, h_{n}\right\}$ equipped with the norm induced from $H$. We write $\left(H_{n},\|\cdot\|_{V}\right)$ when we consider $H_{n}$ equipped with the norm induced from $V$. 
We define by $\Pi_{n}: H \rightarrow H_{n}$ the orthogonal projection of $H$ on $H_{n}$

$$
\Pi_{n} h:=\sum_{i=1}^{n}\left(h, h_{i}\right) h_{i} .
$$

Let $\mathcal{A}_{n}, \mathcal{B}_{n}, \mathcal{G}_{n}: H_{n} \times H_{n} \rightarrow H_{n}$, be defined respectively by

$$
\mathcal{A}_{n} u=\sum_{i=1}^{n}\left\langle\mathcal{A} u, h_{i}\right\rangle h_{i}, \quad \mathcal{B}_{n}(u, v)=\sum_{i=1}^{n}\left\langle\mathcal{B}(u, v), h_{i}\right\rangle h_{i}, \quad \mathcal{G}_{n}(t, u):=\Pi_{n} \mathcal{G}(t, u),
$$

and

$$
X_{n}:=\Pi_{n} X, Y_{n}:=\Pi_{n} Y, a_{0 n}:=\Pi_{n} a_{0}, X_{n}^{M I}(t):=X_{n}\left(t \wedge \mathcal{T}_{M}\right), Y_{n}^{M}(t):=Y_{n}\left(t \wedge \mathcal{T}_{M}\right)
$$

for all $t \in[0, T], v \in H_{n}$ and a.e. $\omega \in \Omega$.

Let $n \in \mathbb{N}$ and $\psi \in \mathcal{L}_{\left(H_{n},\|\cdot\|_{V}\right)}^{2}(\Omega \times[0, T]), \gamma \in \mathcal{L}_{H_{n}}^{2}(\Omega \times[0, T])$. We consider the finite dimensional evolution equations

$\left(P_{n, \psi, \gamma}\right)$

$$
\begin{aligned}
\left(Z_{n, \psi, \gamma}(t), v\right) & +\int_{0}^{t}\left(\mathcal{A}_{n} Z_{n, \psi, \gamma}(s), v\right) \mathrm{d} s=\left(a_{0 n}, v\right) \\
& +\int_{0}^{t}\left(\mathcal{B}_{n}\left(X_{n}(s), Z_{n, \psi, \gamma}(s)\right)+\mathcal{B}_{n}\left(Z_{n, \psi, \gamma}(s), Y_{n}(s)\right), v\right) \mathrm{d} s \\
& +\int_{0}^{t}(\psi(s), v) \mathrm{d} s+\int_{0}^{t}\left(\mathcal{G}_{n}\left(s, Z_{n, \psi, \gamma}(s)\right), v\right) \mathrm{d} w(s) \\
& +\int_{0}^{t}(\gamma(s), v) \mathrm{d} w(s)
\end{aligned}
$$

and for each $M \in \mathbb{N}$ let

$$
\begin{aligned}
&\left(P_{n, \psi, \gamma}^{M}\right) \quad\left(Z_{n, \psi, \gamma}^{M}(t), v\right)+\int_{0}^{t}\left(\mathcal{A}_{n} Z_{n, \psi, \gamma}^{M}(s), v\right) \mathrm{d} s=\left(a_{0 n}, v\right) \\
&+\int_{0}^{t}\left(\mathcal{B}_{n}\left(X_{n}^{M}(s), Z_{n, \psi, \gamma}(s)\right)+\mathcal{B}_{n}\left(Z_{n, \psi, \gamma}(s), Y_{n}^{M}(s)\right), v\right) \mathrm{d} s \\
&+\int_{0}^{t}(\psi(s), v) \mathrm{d} s+\int_{0}^{t}\left(\mathcal{G}_{n}\left(s, Z_{n, \psi, \gamma}^{M}(s)\right), v\right) \mathrm{d} w(s) \\
&+\int_{0}^{t}(\gamma(s), v) \mathrm{d} w(s) \\
& \text { Bereitgestellt von | Technische Universität Berlin } \\
& \text { Angemeldet }
\end{aligned}
$$


for all $t \in[0, T], v \in H_{n}$, and a.e. $\omega \in \Omega$.

\section{THEOREM 4.2}

(i) For each $\Psi \in \mathcal{L}_{V^{*}}^{2}(\Omega \times[0, T]), \Gamma \in \mathcal{L}_{H}^{2}(\Omega \times[0, T])$ there exists $Z_{\Psi . \Gamma} \in \mathcal{L}_{\mathfrak{l}}^{2} \cdot(\Omega \times[0, T])$ satisfying $\left(P_{\Psi, \Gamma}\right)$, and with almost surely continuous trajectories in $H$. The solution is almost surely unique, and there exists a positive constant $c_{1}$ (independent of $a_{0}, \Psi, \Gamma$ ) such that

$$
\begin{aligned}
& \mathrm{E} \sup _{t \in[0, T]} \Delta_{Y}(t)\left\|Z_{\Psi, \Gamma}(t)\right\|^{2}+\mathrm{E} \int_{0}^{T} \Delta_{Y}(t)\left\|Z_{\Psi, \Gamma}(t)\right\|_{V}^{2} \mathrm{~d} s \\
& \leq c_{1}\left[\mathrm{E}\left\|a_{0}\right\|^{2}+\mathrm{E} \int_{0}^{T}\|\Psi(s)\|_{V}^{2} \cdot \mathrm{d} s+\mathrm{E} \int_{0}^{T}\|\Gamma(s)\|^{2} \mathrm{~d} s\right] .
\end{aligned}
$$

(ii) For each $\psi \in \mathcal{L}_{\left(H_{n},\|\cdot\|_{v}\right)}^{2}(\Omega \times[0, T]), \gamma \in \mathcal{L}_{H_{n}}^{2}(\Omega \times[0, T])$ there exists $Z_{n, \psi, \gamma} \in$ $\mathcal{L}_{\left(H_{n},\|\cdot\|_{v)}\right.}^{2}(\Omega \times[0, T])$ satisfying $\left(P_{n, \psi, \gamma}\right)$, and with almost surely continuous trajectories in $H$. The solution is almost surely unique, and there exists a positive constant $c_{2}$ (independent of $n, a_{0}, \psi, \gamma$ ) such that

$$
\begin{aligned}
& \mathrm{E} \sup _{t \in[0, T]} \Delta_{Y}(t)\left\|Z_{n, \psi, \gamma}(t)\right\|^{2}+\mathbf{E} \int_{0}^{T} \Delta_{Y}(t)\left\|Z_{n, \psi, \gamma}(t)\right\|_{V}^{2} \mathrm{~d} s \\
& \leq c_{2}\left[\mathbf{E}\left\|a_{0}\right\|^{2}+\mathbf{E} \int_{0}^{T}\|\psi(s)\|^{2} \mathrm{~d} s+\mathbf{E} \int_{0}^{T}\|\gamma(s)\|^{2} \mathrm{~d} s\right] .
\end{aligned}
$$

Proof. (i) Let $\Psi \in \mathcal{L}_{V \cdot}^{2}(\Omega \times[0, T]), \Gamma \in \mathcal{L}_{H}^{2}(\Omega \times[0, T])$. For each $n \in \mathbb{N}$ let $\Psi_{n}:=$ $\sum_{i=1}^{n}\left\langle\Psi, h_{i}\right\rangle h_{i}, \Gamma_{n}:=\Pi_{n} \Gamma$. For the finite dimensional evolution equation $\left(P_{n, \Psi_{n}, \Gamma_{n}}^{M}\right)$ we apply the theory of finite dimensional Ito equations with Lipschitz continuous nonlinearities (see [12], Theorem 5.5, p. 45). Hence there exists a solution $Z_{n, \Psi_{n}, \Gamma_{n}}^{M} \in$ $\mathcal{L}_{\left(H_{n},\|\cdot\|_{v}\right)}^{2}(\Omega \times[0, T])$ of $\left(P_{n, \Psi_{n}, \Gamma_{n}}^{M}\right)$ and with almost surely continuous trajectories in $H$; this solution is almost surely unique.

For notational simplicity we define $Z_{n}^{M}:=Z_{n, \Psi_{n}}^{M}, \Gamma_{n}$.

Let $M, n \in \mathbb{N}$. Using the Ito formula, the properties of $\mathcal{A}, \mathcal{B}, \mathcal{G}$ we obtain the estimate:

$$
\begin{aligned}
& \mathbf{E} \Delta_{Y_{n}^{M}}(T)\left\|Z_{n}^{M}(T)\right\|^{2}+\mathbf{E} \int_{0}^{T} \Delta_{Y_{n}^{M}}(t)\left\|Z_{n}^{M}(t)\right\|_{V}^{2} \mathrm{~d} t
\end{aligned}
$$

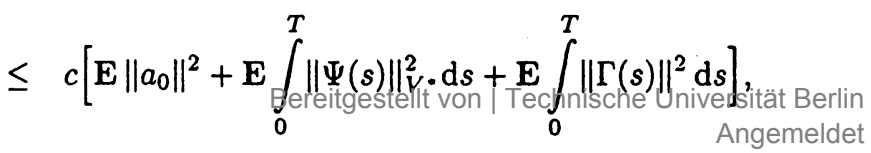


where $c$ is a positive constant independent of $M$ and $n$, but it depends on $\nu, \lambda, T$. We can write

$$
\begin{aligned}
& \mathbf{E} \int_{0}^{T}\left\|Z_{n}^{M I}(t)\right\|_{\nu}^{2} \cdot d t \leq \mathbf{E} \Delta_{Y_{n}^{\prime M}}^{-1}(T) \int_{0}^{T} \Delta_{Y_{n}^{M} M}(t)\left\|Z_{n}^{M T}(t)\right\|_{V}^{2} \cdot d t \\
\leq & c \exp \left\{\frac{b M}{\nu}\right\}\left[\mathbf{E}\left\|a_{0}\right\|^{2}+\mathbf{E} \int_{0}^{T}\|\Psi(t)\|_{\nu}^{2} \cdot \mathrm{d} t+\mathbf{E} \int_{0}^{T}\|\Gamma(t)\|^{2} \mathrm{~d} t\right] .
\end{aligned}
$$

Hence, for fixed $M$ the sequence $\left(Z_{n}^{M I}\right)$ is bounded in the space $\mathcal{L}_{V}^{2} \cdot(\Omega \times[0, T])$. Consequently, there exists a subsequence $\left(n^{\prime}\right)$ of $(n)$ and $Z^{M} \in \mathcal{L}_{V^{*}}^{2}(\Omega \times[0, T])$ such that for $n^{\prime} \rightarrow \infty$ we have

$$
Z_{n^{\prime}}^{M I} \rightarrow Z^{M}
$$

We want to prove that for $n^{\prime} \rightarrow \infty$ the weak convergence $\mathcal{B}_{n^{\prime}}\left(X_{n^{\prime}}^{M}, Z_{n^{\prime}}^{M}\right) \rightarrow \mathcal{B}\left(X^{M}, Z^{M I}\right)$ holds in $\mathcal{L}_{V \cdot}^{2}(\Omega \times[0, T])$. Let $v \in V$ and $v_{n}:=\Pi_{n} v$. We see that

$$
\begin{aligned}
\left(\mathcal{B}_{n}\left(X_{n}^{M}, Z_{n}^{M}\right), v\right)= & \left(\mathcal{B}_{n}\left(X_{n}^{M}, Z_{n}^{M}\right), v_{n}\right)=\left\langle\mathcal{B}\left(X_{n}^{M}, Z_{n}^{M}\right), v_{n}\right\rangle \\
= & \left\langle\mathcal{B}\left(X^{M}, v\right)-\mathcal{B}\left(X_{n}^{M}, v_{n}\right), Z_{n}^{M}\right\rangle+\left\langle\mathcal{B}\left(X^{M}, v\right), Z^{M}-Z_{n}^{M}\right\rangle \\
& +\left\langle\mathcal{B}\left(X^{M}, Z^{M}\right), v\right\rangle .
\end{aligned}
$$

Consequently,

$$
\begin{aligned}
\left\langle\mathcal{B}_{n}\left(X_{n}^{M}, Z_{n}^{M}\right)\right. & \left.-\mathcal{B}\left(X^{M}, Z^{M}\right), v\right\rangle \\
& =\left\langle\mathcal{B}\left(X^{M}, v\right)-\mathcal{B}\left(X_{n}^{M}, v_{n}\right), Z_{n}^{M}\right\rangle+\left\langle\mathcal{B}\left(X^{M}, v\right), Z^{M}-Z_{n}^{M}\right\rangle
\end{aligned}
$$

It holds ${ }^{1}$

$$
\begin{aligned}
& \mathbf{E} \int_{0}^{T}\left\|\mathcal{B}\left(X_{n}^{M}(s), v_{n}\right)-\mathcal{B}\left(X^{M}(s), v\right)\right\|_{V \cdot}^{2} \mathrm{~d} s \\
& \quad \leq b c_{\mathrm{HV}}\left(\|v\|_{V}^{2} \mathrm{E} \int_{0}^{T}\left\|X^{M}(s)-X_{n}^{M}(s)\right\|_{V}^{2} \mathrm{~d} s+\left\|v-v_{n}\right\|_{V}^{2} \mathbf{E} \int_{0}^{T}\left\|X^{M}(s)\right\|_{V}^{2} \mathrm{~d} s\right) .
\end{aligned}
$$

Since $v_{n}$ and $X_{n}^{M}$ are the Fourier expansions of $v$ and $X^{M}$, respectively, it follows that

$$
\lim _{n \rightarrow \infty} \mathbf{E} \int_{0}^{T}\left\|\mathcal{B}\left(X_{n}^{M}(s), v_{n}\right)-\mathcal{B}\left(X^{M}(s), v\right)\right\|_{V \cdot \mathrm{d} s}^{2}=0
$$

Using (5), (7) in (6) we get 


$$
\lim _{n^{\prime} \rightarrow \infty} \mathbf{E} \int_{0}^{T}\left(\mathcal{B}_{n^{\prime}}\left(X_{n^{\prime}}^{M}(s), Z_{n^{\prime}}^{M I}(s)\right), \xi(s)\right) \mathrm{d} s=\mathbf{E} \int_{0}^{T}\left\langle\mathcal{B}\left(X^{M}(s), Z^{M}(s)\right), \xi(s)\right\rangle \mathrm{d} s
$$

for all $\xi \in \mathcal{D}_{V}(\Omega \times[0, T])$. Since $\mathcal{B}_{n^{\prime}}\left(X_{n^{\prime}}^{M I}, Z_{n^{\prime}}^{M I}\right), \mathcal{B}\left(X^{M}, Z^{M I}\right) \in \mathcal{L}_{V}^{2} \cdot(\Omega \times[0, T])$ and $\mathcal{D}_{V}(\Omega \times[0, T])$ is dense in $\mathcal{L}_{\mathrm{l}}^{2} \cdot(\Omega \times[0, T])$, we have $\mathcal{B}_{n^{\prime}}\left(X_{n^{\prime}}^{M I}, Z_{n^{\prime}}^{M}\right) \rightarrow \mathcal{B}\left(X^{M I}, Z^{M I}\right)$ for $n^{\prime} \rightarrow \infty$. Analogously we can prove that $\mathcal{B}_{n^{\prime}}\left(Z_{n^{\prime}}^{M}, Y_{n^{\prime}}^{M}\right) \rightarrow \mathcal{B}\left(Z^{M I}, Y^{M}\right)$ for $n^{\prime} \rightarrow \infty$.

We take the limit $n^{\prime} \rightarrow \infty$ in $\left(P_{n^{\prime}, \Psi_{n^{\prime}}, \Gamma_{n^{\prime}}}^{M}\right)$, use the weak convergence (5), as soon as the strong convergences of $\left(X_{n}^{M}\right)$ to $X^{M}$ and of $\left(Y_{n}^{M}\right)$ to $Y^{M}$ in the space $\mathcal{L}_{H}^{2}(\Omega \times[0, T])$ and a well-known result on weak convergence (see [20] Proposition 21.27, p. 261) to obtain

$$
\begin{aligned}
\left(Z^{M}(t), v\right)= & \left(a_{0}, v\right)-\int_{0}^{t}\left\langle\mathcal{A} Z^{M}(s), v\right\rangle \mathrm{d} s+\int_{0}^{t}\left\langle\mathcal{B}\left(X^{M}(s), Z^{M}(s)\right)\right. \\
& \left.+\mathcal{B}\left(Z^{M}(s), Y^{M}(s)\right), v\right\rangle \mathrm{d} s+\int_{0}^{t}\langle\Psi(s), v\rangle \mathrm{d} s \\
& +\int_{0}^{t}\left(\mathcal{G}\left(s, Z^{M}(s)\right), v\right) \mathrm{d} w(s)+\int_{0}^{t}(\Gamma(s), v) \mathrm{d} w(s)
\end{aligned}
$$

for all $v \in V$ and $P \times \Lambda$ a.e. $(\omega, t) \in \Omega \times[0, T]$. The process appearing in the right side of (8) has a continuous modification (as an $H$ valued process), and this process we identify with $\left(Z_{\Psi, \Gamma}^{M I}(t)\right)_{t \in[0, T]}$ (see [13], Theorem 2, p. 73). So, $\left(Z_{\Psi, \Gamma}^{M I}(t)\right)_{t \in[0, T]}$ is a process from the space $\mathcal{L}_{V}^{2}(\Omega \times[0, T])$ which has almost surely continuous trajectories in $H$ and satisfies $\left(P_{\Psi, \Gamma}^{M I}\right)$ (identically with (8)) for all $v \in V, t \in[0, T]$ and a.e. $\omega \in \Omega$. By standard methods (see the final part of the proof) we can prove that the solution of $\left(P_{\Psi, \Gamma}^{M}\right)$ is almost surely unique.

Let $\Omega_{K}$ be the set of all $\omega \in \Omega$ such that $Z_{\Psi, \Gamma}^{K}(\omega, \cdot)$ satisfies $\left(P_{\Psi, \Gamma}^{K}\right)$ for all $t \in[0, T], v \in$ $V$ and such that $Z_{\Psi, \Gamma}^{K}(\omega, \cdot)$ has continuous trajectories in $H$. We define $\Omega^{\prime}:=\bigcap_{K=1}^{\infty} \Omega_{K}$.

We also consider

$$
S:=\bigcup_{M=1}^{\infty} \bigcup_{1 \leq K \leq M}\left\{\omega \in \Omega^{\prime} \mid \mathcal{T}_{K}=T \text { and } \exists t \in[0, T]: Z_{\Psi, \Gamma}^{K}(\omega, t) \neq Z_{\Psi, \Gamma}^{M}(\omega, t)\right\} .
$$

We have $\mathbf{P}(S)=0$, because otherwise there exist two natural numbers $M_{0}, K_{0}$ with $K_{0}<M_{0}$ such that the set

$$
S_{M_{0}, K_{0}}:=\left\{\omega \in \Omega^{\prime} \mid \mathcal{T}_{K_{0}}=T \text { and } \exists t \in[0, T]: Z_{\Psi, \Gamma}^{K_{0}}(\omega, t) \neq Z_{\Psi, \Gamma}^{M_{0}}(\omega, t)\right\}
$$

has the measure $\mathbf{P}\left(S_{M_{0}, K_{0}}\right)>0$. We define for each $t \in[0, T]$

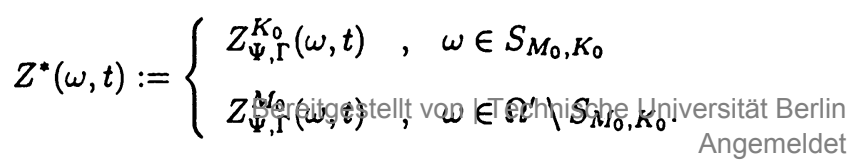


We see that for all $\omega \in S_{M_{0}, K_{0}}$ there exists $t \in[0, T]$ such that $Z^{*}(\omega, t) \neq Z^{M_{0}}(\omega, t)$. This contradicts to the almost surely uniqueness of the solution of $\left(P_{\Psi, \Gamma}^{M_{0}}\right)$. Consequently, $\mathbf{P}(S)=0$.

We define

$$
\Omega^{\prime \prime}:=\bigcup_{M=1}^{\infty}\left\{\mathcal{T}_{M I}=T\right\} .
$$

Obviously $\mathbf{P}\left(\left(\Omega^{\prime} \cap \Omega^{\prime \prime}\right) \backslash S\right)=1$ (see also (2)). Let $\omega \in\left(\Omega^{\prime} \cap \Omega^{\prime \prime}\right) \backslash S$. For this $\omega$ there exists a natural number $M_{0}$ such that $\mathcal{T}_{M}(\omega)=T$ for all $M \geq M_{0}$. Hence $X^{M}(s)=X(s)$ and $Y^{M I}(s)=Y(s)$ for all $s \in[0, T]$ and for all $M \geq M_{0}$. Equation $\left(P_{\Psi, \Gamma}^{M}\right)$ implies

$$
\begin{aligned}
\left(Z_{\Psi, \Gamma}^{M}(t), v\right) & +\int_{0}^{t}\left\langle\mathcal{A} Z_{\Psi, \Gamma}^{M}(s), v\right\rangle \mathrm{d} s=\left(a_{0}, v\right) \\
& +\int_{0}^{t}\left\langle\mathcal{B}\left(X(s), Z_{\Psi, \Gamma}^{M}(s)\right)+\mathcal{B}\left(Z_{\Psi, \Gamma}^{M}(s), Y(s)\right), v\right\rangle \mathrm{d} s \\
& +\int_{0}^{t}\langle\Psi(s), v\rangle \mathrm{d} s+\int_{0}^{t}\left(\mathcal{G}\left(s, Z_{\Psi, \Gamma}^{M}(s)\right), v\right) \mathrm{d} w(s)+\int_{0}^{t}(\Gamma(s), v) \mathrm{d} w(s)
\end{aligned}
$$

for all $M \geq M_{0}$ and all $t \in[0, T], v \in V$. We have

$$
\lim _{M \rightarrow \infty} \int_{0}^{T}\left\|Z_{\Psi, \Gamma}^{M}(t)-Z_{\Psi, \Gamma}^{M_{0}}(t)\right\|_{V}^{2} \mathrm{~d} t=0
$$

and

$$
\lim _{M \rightarrow \infty} \sup _{t \in[0, T]}\left\|Z_{\Psi, \Gamma}^{M}(t)-Z_{\Psi, \Gamma}^{M T_{0}}(t)\right\|^{2}=0
$$

For each $t \in[0, T]$ we define

$$
Z_{\Psi, \Gamma}(\omega, t):=Z_{\Psi, \Gamma}^{M_{0}}(\omega, t)=\lim _{M \rightarrow \infty} Z_{\Psi, \Gamma}^{M}(\omega, t) .
$$

This definition is correct because $\omega \notin S$. Then (9) implies

$$
\begin{aligned}
&\left(Z_{\Psi, \Gamma}(t), v\right)+\int_{0}^{t}\left(\mathcal{A} Z_{\Psi, \Gamma}(s), v\right) \mathrm{d} s=\left(a_{0}, v\right) \\
&+\int_{0}^{t}\left\langle\mathcal{B}\left(X(s), Z_{\Psi, \Gamma}(s)\right)+\mathcal{B}\left(Z_{\Psi, \Gamma}(s), Y(s)\right), v\right\rangle \mathrm{d} s \\
&+\int_{0}^{t}\langle\Psi(s), v\rangle \mathrm{d} s+\int_{\text {Be } i \text { itgestellt von } \mid \text { Technische Uniqersität Berlin }}^{t}\left(\mathcal{G}\left(s, Z_{\Psi, \Gamma}(s)\right), v\right) \mathrm{d} w(s)+\int_{\text {Angemeldet }}^{t}(\Gamma(s), v) \mathrm{d} w(s) \\
& \text { Heruntergeladen am | 04.10.18 15:22 }
\end{aligned}
$$


for all $\omega \in\left(\Omega \cap \Omega^{\prime \prime}\right) \backslash S, t \in[0, T], v \in V$. The process $\left(Z_{\Psi, \Gamma}(t)\right)_{t \in[0, T]}$ is $V$-valued, $\mathcal{F} \times \mathcal{B}_{[0, T]}$-measurable, adapted to the filtration $\left(\mathcal{F}_{t}\right)_{t \in[0, T]}$ and has almost surely continuous trajectories in $H$, because all $Z_{\Psi, \Gamma}^{M}$ have this properties. For $Z_{\Psi, \Gamma}^{M}$ we can prove an analogous inequality as (3). Thus we get

$$
\begin{aligned}
\mathbf{E} \Delta_{Y}(T)\left\|Z_{\Psi, \Gamma}(T)\right\|^{2}+ & \mathbf{E} \int_{0}^{T} \Delta_{Y}(t)\left\|Z_{\Psi, \Gamma}(t)\right\|_{V}^{2} \mathrm{~d} t \\
\leq & \liminf _{M \rightarrow \infty}\left\{\mathbf{E} \Delta_{Y^{M}}(T)\left\|Z_{\Psi, \Gamma}^{M}(T)\right\|^{2}\right. \\
& \left.+\mathbf{E} \int_{0}^{T} \Delta_{Y^{M}}(t)\left\|Z_{\Psi, \Gamma}^{M I}(t)\right\|_{V}^{2} \mathrm{~d} t\right\} \\
\leq & c\left[\mathbf{E}\left\|a_{0}\right\|^{2}+\mathbf{E} \int_{0}^{T}\|\Psi(s)\|_{V}^{2} \mathrm{~d} s+\mathbf{E} \int_{0}^{T}\|\Gamma(s)\|^{2} \mathrm{~d} s\right]
\end{aligned}
$$

where $c$ is the same constant as in (3).

Now we prove that equation $\left(P_{\Psi, \Gamma}\right)$ has an almost surely unique solution. Let

$$
e_{1}(t):=\Delta_{Y}(t) \exp \{-\lambda t\}
$$

We assume that $\dot{Z}$ and $Z$ are two solutions of $\left(P_{\Psi, \Gamma}\right)$ which have almost surely continuous trajectories in $H$. Then by the Ito formula and by the properties of $\mathcal{A}, \mathcal{B}$ and $\mathcal{G}$ we have for each $t \in[0, T]$ and a.e. $\omega \in \Omega$

$$
\begin{aligned}
e_{1}(t)\|\tilde{Z}(t)-Z(t)\|^{2} & +\nu \int_{0}^{t} e_{1}(s)\|\tilde{Z}(s)-Z(s)\|_{V}^{2} \mathrm{~d} s \\
& \leq 2 \int_{0}^{t} e_{1}(s)(\mathcal{G}(s, \tilde{Z}(s))-\mathcal{G}(s, Z(s)), \tilde{Z}(s)-Z(s)) \mathrm{d} w(s) .
\end{aligned}
$$

This implies

$$
\mathbf{E} \int_{0}^{T} \Delta_{Y}(s)\|\tilde{Z}(s)-Z(s)\|_{V}^{2} \mathrm{~d} s=0
$$

Hence $\tilde{Z}(\omega, t)=Z(\omega, t)$ for $P \times \Lambda$ a.e. $(\omega, t) \in \Omega \times[0, T]$. Using this result and (12) we deduce that

$$
\mathrm{E} \sup _{t \in[0, T]} \Delta_{Y}(t)\|\tilde{Z}(t)-Z(t)\|^{2}=0,
$$

which means that $\left(P_{\Psi, \Gamma}\right)$ has an almost surely unique solution.

(ii) The existence, estimation, and (almost surely) uniqueness of the solution $Z_{n, \psi, \gamma}$ of $\left(P_{n, \psi, \gamma}\right)$ can be proved analogouslfyetitthetoltoof of $($ (i) $)$. nische Universität Berlin 
Other properties of the solution of equation $\left(P_{\Psi, \Gamma}\right)$ are contained in the next lemma. This results have nice applications in the investigation of the control problem concerning the stochastic Navier-Stokes equation, more precisely in the proof of the existence of optimal controls and in the formulation of a stochastic minimum principle (see [2], [11]).

LEMMA 4.3 We assume that $\mathrm{E} \Delta_{Y}^{-2}(T)<\infty$. Let $\left(\psi_{n}\right),\left(\gamma_{n}\right)$ be sequences in

$\mathcal{L}_{V}^{2}(\Omega \times[0, T])$ and $\mathcal{L}_{H}^{2}(\Omega \times[0, T])$, respectively, such that $\psi_{n} \in \mathcal{L}_{\left(H_{n},\|\cdot\|_{V}\right)}^{2}(\Omega \times[0, T]), \gamma_{n} \in$ $\mathcal{L}_{H_{n}}^{2}(\Omega \times[0, T])$ for each $n \in \mathbb{N}$. If $\left(\mathrm{J} \psi_{n}\right)$ converges weakly to $\Psi \in \mathcal{L}_{V}^{2} \cdot(\Omega \times[0, T])$ and $\left(\gamma_{n}\right)$ converges weakly to $\Gamma \in \mathcal{L}_{H}^{2}(\Omega \times[0, T])$, then for $n \rightarrow \infty$ we have

$$
\Delta_{Y} Z_{n, \psi_{n}, \gamma_{n}} \rightarrow \Delta_{Y} Z_{\Psi, \Gamma} \quad \text { in } \quad \mathcal{L}_{V}^{2}(\Omega \times[0, T])
$$

and

$$
\Delta_{Y}(T) Z_{n, \psi_{n}, \gamma_{n}}(T) \rightarrow \Delta_{Y}(T) Z_{\Psi, \Gamma}(T) \quad \text { in } \quad \mathcal{L}_{H}^{2}(\Omega)
$$

Proof. Because $\left(\mathrm{J} \psi_{n}\right)$ converges weakly to $\Psi \in \mathcal{L}_{V \cdot}^{2}(\Omega \times[0, T])$ and $\left(\gamma_{n}\right)$ converges weakly to $\Gamma \in \mathcal{L}_{H}^{2}(\Omega \times[0, T])$, it follows that there exists a constant $c_{3}>0$ such that for all $n \in \mathbb{N}$

$$
\mathbf{E} \int_{0}^{T}\left\|\psi_{n}(t)\right\|_{V}^{2} \mathrm{~d} t+\mathbf{E} \int_{0}^{T}\left\|\gamma_{n}(t)\right\|^{2} \mathrm{~d} t \leq c_{3} .
$$

For simplicity we define $Z_{n}:=Z_{n, \psi_{n}, \gamma_{n}}$ and $Z_{n}^{M}:=Z_{n, \psi_{n}, \gamma_{n}}^{M}$. Applying Theorem 4.2 we obtain

$$
\begin{array}{r}
\sup _{1 \leq n}\left\{\mathbf{E} \Delta_{Y}(T)\left\|Z_{n}(T)-Z_{\Psi, \Gamma}(T)\right\|^{2}+\mathbf{E} \int_{0}^{T} \Delta_{Y}(s)\left\|Z_{n}(s)-Z_{\Psi, \Gamma}(s)\right\|_{V}^{2} \mathrm{~d} s\right\} \\
\leq\left(c_{1}+c_{2}\right)\left(c_{3}+\mathbf{E}\left\|a_{0}\right\|^{2}+\mathbf{E} \int_{0}^{T}\|\Psi(s)\|_{V}^{2} \cdot \mathrm{d} s+\mathbf{E} \int_{0}^{T}\|\Gamma(s)\|^{2} \mathrm{~d} s\right) .
\end{array}
$$

Let $\xi \in \mathcal{D}_{V \cdot}(\Omega \times[0, T])$. Next we will prove that

$$
\lim _{n \rightarrow \infty} \mathbf{E} \int_{0}^{T}\left\langle\xi(s), Z_{n}(s)-Z_{\Psi, \Gamma}(s)\right\rangle \mathrm{d} s=0
$$

Since $\lim _{M \rightarrow \infty} \mathcal{T}_{M}=T$ (see (1)) for a.e. $\omega \in \Omega, \mathbf{E} \Delta_{Y}^{-2}(T)<\infty$ and $\xi \in \mathcal{D}_{V} \cdot(\Omega \times[0, T])$, we get

$$
\lim _{M \rightarrow \infty} \mathbf{E} \int_{\tau_{M}}^{T} \Delta_{Y}^{-1}(s)\|\xi(s)\|_{V}^{2} \cdot \mathrm{d} s=0 .
$$

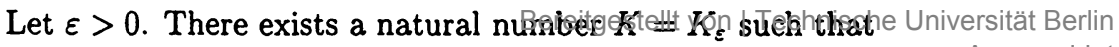




$$
\sup _{1 \leq n}\left\{\mathrm{E} \int_{0}^{T} \Delta_{Y}(s)\left\|Z_{n}(s)-Z_{\Psi, \Gamma}(s)\right\|_{V}^{2} \mathrm{~d} s\right\} \mathrm{E} \int_{\mathcal{T}_{K}}^{T} \Delta_{Y}^{-1}(s)\|\xi(s)\|_{i}^{2} . \mathrm{d} s<\frac{\varepsilon^{2}}{4}
$$

Relation (14) implies

$$
\begin{aligned}
& \mathbf{E} \int_{\mathcal{T}_{K}}^{T}\left\langle\xi(s), Z_{n}(s)-Z_{\Psi, \Gamma}(s)\right\rangle \mathrm{d} s \\
& \leq\left(\sup _{1 \leq n}\left\{\mathbf{E} \int_{0}^{T} \Delta_{Y}(s)\left\|Z_{n}(s)-Z_{\Psi, \Gamma}(s)\right\|_{V}^{2} \mathrm{~d} s\right\} \mathbf{E} \int_{\mathcal{T}_{K}}^{T} \Delta_{Y}^{-1}(s)\|\xi(s)\|_{V}^{2} \cdot \mathrm{d} s\right)^{1 / 2}<\frac{\varepsilon}{2}
\end{aligned}
$$

for all $n \in \mathbb{N}$.

From the (almost surely) uniqueness of the solutions of $\left(P_{\Psi, \Gamma}^{K}\right)$ and $\left(P_{n, \psi_{n}, \gamma_{n}}^{K}\right)$, respectively, we conclude that

$$
\mathbf{E} \int_{0}^{\tau_{K}}\left\|Z_{n}(s)-Z_{n}^{K}(s)\right\|_{V}^{2} \mathrm{~d} s=\mathbf{E} \int_{0}^{\tau_{K}}\left\|Z_{\Psi, \Gamma}^{K}(s)-Z_{\Psi, \Gamma}(s)\right\|_{V}^{2} \mathrm{~d} s=0 .
$$

Then for all $n \in \mathbb{N}$ we have

$$
\begin{aligned}
& \left|\mathbf{E} \int_{0}^{T}\left\langle\xi(s), Z_{n}(s)-Z_{\Psi, \Gamma}(s)\right\rangle \mathrm{d} s\right| \\
\leq & \left|\mathbf{E} \int_{0}^{\tau_{K}}\left\langle\xi(s), Z_{n}(s)-Z_{\Psi, \Gamma}(s)\right\rangle \mathrm{d} s\right|+\left|\mathbf{E} \int_{\tau_{K}}^{T}\left\langle\xi(s), Z_{n}(s)-Z_{\Psi, \Gamma}(s)\right\rangle \mathrm{d} s\right| \\
\leq & \left|\mathbf{E} \int_{0}^{\tau_{K}}\left\langle\xi(s), Z_{n}(s)-Z_{n}^{K}(s)\right\rangle \mathrm{d} s\right|+\left|\mathbf{E} \int_{0}^{\tau_{K}}\left\langle\xi(s), Z_{n}^{K}(s)-Z_{\Psi, \Gamma}^{K}(s)\right\rangle \mathrm{d} s\right| \\
& +\left|\mathbf{E} \int_{0}^{\tau_{K}}\left\langle\xi(s), Z_{\Psi, \Gamma}^{K}(s)-Z_{\Psi, \Gamma}(s)\right\rangle \mathrm{d} s\right|+\frac{\varepsilon}{2} \\
= & \left|\mathbf{E} \int_{0}^{\tau_{K}}\left\langle\xi(s), Z_{n}^{K}(s)-Z_{\Psi, \Gamma}^{K}(s)\right\rangle \mathrm{d} s\right|+\frac{\varepsilon}{2} .
\end{aligned}
$$

In the following we prove that there exists an $n_{\varepsilon}>0$ such that

$$
\left|\mathrm{E} \int_{0}^{\tau_{K}}\left\langle\xi(s), Z_{n}^{K}(s)-Z_{\Psi, \Gamma}^{K}(s)\right\rangle \mathrm{d} s\right|<\frac{\varepsilon}{2}
$$

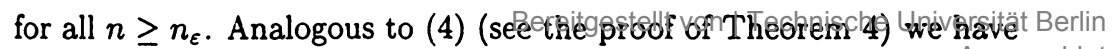




$$
\mathbf{E} \int_{0}^{T}\left\|Z_{n}^{K}(s)\right\|_{V}^{2} \mathrm{~d} s \leq c \exp \left\{\frac{b K}{\nu}\right\}\left[\mathbf{E}\left\|a_{0}\right\|^{2}+c_{3}\right] .
$$

Hence $\left(Z_{n}^{K}\right)$ is a bounded sequence from $\mathcal{L}_{V}^{2}(\Omega \times[0, T])$. Consequently, by sequential weak compactness there exists a subsequence $\left(n^{\prime}\right)$ of $(n)$ and $Z^{K} \in \mathcal{L}_{V}^{2}(\Omega \times[0, T])$ such that for $n^{\prime} \rightarrow \infty$ we have

$$
Z_{n^{\prime}}^{K} \rightarrow Z^{K} \quad \text { in } \mathcal{L}_{V}^{2}(\Omega \times[0, T]) .
$$

As in the proof of Theorem 4.2 we can show that $\mathcal{B}_{n^{\prime}}\left(X_{n^{\prime}}^{K}, Z_{n^{\prime}}^{K}\right) \rightarrow \mathcal{B}\left(X^{K}, Z^{K}\right)$ and $\mathcal{B}_{n^{\prime}}\left(Z_{n^{\prime}}^{K}, Y_{n^{\prime}}^{K}\right) \rightarrow \mathcal{B}\left(Z^{K}, Y^{K}\right)$ in $\mathcal{L}_{V \cdot}^{2}(\Omega \times[0, T])$ for $n^{\prime} \rightarrow \infty$. We take the limit $n^{\prime} \rightarrow \infty$ in equation $\left(P_{n^{\prime}, \psi_{n^{\prime}}, \gamma_{n^{\prime}}}^{K}\right)$, use the weak convergences given in the hypothesis and in (17), then the strong convergences of $\left(X_{n^{\prime}}^{K}\right)$ to $X^{K}$ and of $\left(Y_{n^{\prime}}^{K}\right)$ to $Y^{K}$ in the space $\mathcal{L}_{H}^{2}(\Omega \times[0, T])$ and Proposition 21.27 from p. 261 in [20]. Then we obtain

$$
\begin{aligned}
& \left(Z^{K}(t), v\right)+\int_{0}^{t}\left\langle\mathcal{A} Z^{K}(s), v\right\rangle \mathrm{d} s \\
= & \left(a_{0}, v\right)+\int_{0}^{t}\left\langle\mathcal{B}\left(X^{K}(s), Z^{K}(s)\right)+\mathcal{B}\left(Z^{K}(s), Y^{K}(s)\right), v\right\rangle \mathrm{d} s \\
+ & \int_{0}^{t}\langle\Psi(s), v\rangle \mathrm{d} s+\int_{0}^{t}\left(\mathcal{G}\left(s, Z^{K}(s)\right), v\right) \mathrm{d} w(s)+\int_{0}^{t}(\Gamma(s), v) \mathrm{d} w(s)
\end{aligned}
$$

for all $v \in V$ and $P \times \Lambda$ a.e. $(\omega, t) \in \Omega \times[0, T]$. The (almost surely) uniqueness of the solution of equation $\left(P_{\Psi, \Gamma}^{K}\right)$ implies that

$$
Z^{K}(\omega, t)=Z_{\Psi, \Gamma}^{K}(\omega, t) \quad \text { for } P \times \Lambda \quad \text { a.e. } \quad(\omega, t) \in \Omega \times[0, T] .
$$

Hence

$$
Z_{n^{\prime}}^{K} \rightarrow Z_{\Psi, \Gamma}^{K} \quad \text { in } \quad \mathcal{L}_{V}^{2}(\Omega \times[0, T])
$$

We also see that each weakly convergent subsequence of $\left(Z_{n}^{K}\right)$ converges weakly to the same limit $Z_{\Psi, \Gamma}^{K}$. Therefore, the whole sequence $\left(Z_{n}^{K}\right)$ converges weakly to $Z_{\Psi, \Gamma}^{K}$ in $\mathcal{L}_{V}^{2}(\Omega \times[0, T])$ (see [19] Proposition 10.13, p. 480).

Hence, there exists $n_{\varepsilon}>0$ such that for all $n \geq n_{\varepsilon}$ we have

$$
\mathbf{E} \int_{0}^{\tau_{K}}\left\langle\xi(s), Z_{n}^{K}(s)-Z_{\Psi, \Gamma}^{K}(s)\right\rangle \mathrm{d} s<\frac{\varepsilon}{2}
$$

Using (16) we deduce that

$$
\left|\mathrm{E} \int_{0}^{T}\left\langle\xi(s), Z_{n}(s)-Z_{\Psi, \Gamma}(s)\right\rangle \mathrm{d} s\right|<\varepsilon \text { for all } n \geq n_{\varepsilon}
$$


and consequently,

$$
\lim _{n \rightarrow \infty} \mathbf{E} \int_{0}^{T}\left\langle\xi(s), Z_{n}(s)-Z_{\Psi, \Gamma}(s)\right\rangle \mathrm{d} s=0 .
$$

Because $\mathcal{D}_{V} \cdot(\Omega \times[0, T])$ is dense in $\mathcal{L}_{V}^{2} \cdot(\Omega \times[0, T])$ and $\Delta_{Y} Z_{n}, \Delta_{Y} Z_{\Psi, \Gamma} \in \mathcal{L}_{\vec{l}}^{2} \cdot(\Omega \times[0, T])$ (we do not know whether $Z_{n}, Z_{\Psi, \Gamma} \in \mathcal{L}_{V}^{2}(\Omega \times[0, T])$ ) we conclude that for $n \rightarrow \infty$

$$
\Delta_{Y} Z_{n} \rightarrow \Delta_{Y} Z_{\Psi, \Gamma} \quad \text { in } \quad \mathcal{L}_{V}^{2}(\Omega \times[0, T]) .
$$

We want to prove that for all $\xi \in \mathcal{D}_{V}(\Omega)$ we have

$$
\lim _{n \rightarrow \infty} \mathbf{E}\left(Z_{\Psi, \Gamma}(T)-Z_{n}(T), \xi\right)=0
$$

Let $\xi=v \phi \in \mathcal{D}_{V}(\Omega), \varepsilon>0$. There exists an index $K \in \mathbb{N}$ such that for all $n \in \mathbb{N}$ we get

$$
\begin{aligned}
& \left|\mathbf{E}\left(Z_{\Psi, \Gamma}(T)-Z_{n}(T), v\right) \phi\right| \leq\left|\mathbf{E}\left(Z_{\Psi, \Gamma}(T)-Z_{n}(T), \Pi_{K} v\right) \phi\right| \\
& +\left\|v-\Pi_{K} v\right\|\left\{\mathbf{E}\left(\phi^{2} \Delta_{Y}^{-1}(T)\right) \sup _{1 \leq n}\left[\mathbf{E} \Delta_{Y}(T)\left\|Z_{\Psi, \Gamma}(T)-Z_{n}(T)\right\|^{2}\right]\right\}^{1 / 2} \\
\leq & \left|\mathbf{E}\left(Z_{\Psi, \Gamma}(T)-Z_{n}(T), \Pi_{K} v\right) \phi\right|+\frac{\varepsilon}{2} .
\end{aligned}
$$

In the second inequality we have used (13). From $\left(P_{n, \psi_{n}}\right)$ and $\left(P_{\Psi, \Gamma}\right)$ we conclude that

$$
\begin{aligned}
& \mathbf{E} \phi\left(Z_{\Psi, \Gamma}(T)-Z_{n}(T), \Pi_{K} v\right)+\mathbf{E} \phi \int_{0}^{T}\left\langle\mathcal{A} Z_{\Psi, \Gamma}(s)-\mathcal{A} Z_{n}(s), \Pi_{K} v\right\rangle \mathrm{d} s \\
& =\mathbf{E} \phi \int_{0}^{T}\left\langle\mathcal{B}\left(X(s), Z_{\Psi, \Gamma}(s)\right)-\mathcal{B}\left(X_{n}(s), Z_{n}(s)\right)+\mathcal{B}\left(Z_{\Psi, \Gamma}(s), Y(s)\right)\right. \\
& \left.-\mathcal{B}\left(Z_{n}(s), Y_{n}(s)\right), \Pi_{K} v\right\rangle \mathrm{d} s \\
& +\mathbf{E} \phi \int_{0}^{T}\left\langle\Psi(s)-\mathrm{J} \psi_{n}(s), \Pi_{K} v\right\rangle \mathrm{d} s+\mathbf{E} \phi \int_{0}^{T}\left(\mathcal{G}\left(s, Z_{\Psi, \Gamma}(s)-Z_{n}(s)\right), \Pi_{K} v\right) \mathrm{d} w(s) \\
& +\mathbf{E} \phi \int_{0}^{T}\left(\Gamma(s)-\gamma_{n}(s), \Pi_{K} v\right) \mathrm{d} w(s) .
\end{aligned}
$$

In the above equation we take the limit $n \rightarrow \infty$, use the weak convergence (18) and obtain that there exists an $n_{\varepsilon}>0$ such that

$$
\left|\mathrm{E}\left(Z_{\Psi, \Gamma}(T)-Z_{n}(T), \Pi_{K} v\right) \phi\right|<\frac{\varepsilon}{2} \text { for all } n \geq n_{\varepsilon}
$$

We use (20) to deduce that 
Hence (19) yields. Since $\mathcal{D}_{V}(\Omega)$ is dense in $\mathcal{L}_{V}^{2}(\Omega) \hookrightarrow \mathcal{L}_{H}^{2}(\Omega)$ and since we have $\Delta_{Y}(T) Z_{n}(T), \Delta_{Y}(T) Z_{\Psi, \Gamma}(T) \in \mathcal{L}_{H}^{2}(\Omega)$ (note, we do not know whether $Z_{n}(T), Z_{\Psi, \Gamma}(T) \in$ $\left.\mathcal{L}_{H}^{2}(\Omega)\right)$ we conclude that

$$
\Delta_{Y}(T) Z_{n}(T) \rightarrow \Delta_{Y}(T) Z_{\Psi, \Gamma}(T) \quad \text { in } \quad \mathcal{L}_{H}^{2}(\Omega)
$$

Lemma 4.3 is proved.

Remark 4.4

1) Theorem 4.2 hold also under weaker assumptions on $\mathcal{G}$, namely, $\mathcal{G}(t, \cdot)$ must not be linear. Of course then the proof of the theorem must be changed.

2) If in the above lemma we assume that

$$
\mathbf{E} \int_{0}^{T}\|\Psi(t)\|_{V}^{4} \cdot \mathrm{d} t<\infty, \quad \mathbf{E} \int_{0}^{T}\|\Gamma(t)\|^{4} \mathrm{~d} t<\infty
$$

and for all $n \in \mathbb{N}$

$$
\mathbf{E} \int_{0}^{T}\left\|\psi_{n}(t)\right\|^{4} \mathrm{~d} t<\infty, \mathbf{E} \int_{0}^{T}\left\|\gamma_{n}(t)\right\|^{4} \mathrm{~d} t<\infty,
$$

then for $n \rightarrow \infty$ we have

$$
\begin{aligned}
\mathbf{E} \sup _{t \in[0, T]} \Delta_{Y}^{2}(t)\left\|Z_{\Psi, \Gamma}(t)\right\|^{4} & +\mathbf{E}\left(\int_{0}^{T} \Delta_{Y}(t)\left\|Z_{\Psi, \Gamma}(t)\right\|_{V}^{2} \mathrm{~d} s\right)^{2} \\
& \leq c_{1}\left[\mathbf{E}\left\|a_{0}\right\|^{4}+\mathbf{E} \int_{0}^{T}\|\Psi(s)\|_{V^{*}}^{4} \mathrm{~d} s+\mathbf{E} \int_{0}^{T}\|\Gamma(s)\|^{4} \mathrm{~d} s\right]
\end{aligned}
$$

and

$$
\begin{aligned}
\mathbf{E} \sup _{t \in[0, T]} \Delta_{Y}^{2}(t)\left\|Z_{n, \psi, \gamma}(t)\right\|^{4} & +\mathbf{E}\left(\int_{0}^{T} \Delta_{Y}(t)\left\|Z_{n, \psi, \gamma}(t)\right\|_{V}^{2} \mathrm{~d} s\right)^{2} \\
& \leq c_{1}\left[\mathbf{E}\left\|a_{0}\right\|^{4}+\mathbf{E} \int_{0}^{T}\|\psi(s)\|^{4} \mathrm{~d} s+\mathbf{E} \int_{0}^{T}\|\gamma(s)\|^{4} \mathrm{~d} s\right] .
\end{aligned}
$$

This estimates one can deduce by using the Ito formula and properties of the stochastic integral (for more details see [2], Proposition B.2). We also can prove that

$$
Z_{n, \psi_{n}, \gamma_{n}} \rightarrow Z_{\Psi, \Gamma} \quad \text { in } \quad \mathcal{L}_{V}^{2}(\Omega \times[0, T])
$$

and

$$
Z_{n, \psi_{n}, \gamma_{n}}(T) \rightarrow Z_{\Psi, \Gamma}(T) \text { in } \quad \mathcal{L}_{H}^{2}(\Omega) .
$$

This stronger convergences hold, because $Z_{n, \psi_{n}, \gamma_{n}}, Z_{\Psi, \Gamma} \in \mathcal{L}_{V}^{2}(\Omega \times[0, T])$ and $Z_{n, \psi_{n}, \gamma_{n}}(T)$, $Z_{\Psi, \Gamma}(T) \in \mathcal{L}_{H}^{2}(\Omega)$. 


\section{LINEAR APPROXIMATION OF THE SOLUTION OF THE STOCHASTIC NAVIER- STOKES EQUATION}

In this section we show how equations of the type $\left(P_{\Psi, \Gamma}\right)$ can be used to develop a linear approximation method for the stochastic Navier-Stokes equation (21).

Before we give the definition for the solution of the stochastic Navier-Stokes equation we need some additional hypothesis:

(ix) $\mathcal{C}:[0, T] \times H \rightarrow H$ is a mapping such that

(a) $\|\mathcal{C}(t, u)-\mathcal{C}(t, v)\|^{2} \leq \lambda\|u-v\|^{2}$ for all $t \in[0, T], u, v \in H$;

(b) $\mathcal{C}(t, 0)=0$ for all $t \in[0, T]$;

(c) $\mathcal{C}(\cdot, v) \in \mathcal{L}_{H}^{2}[0, T]$ for all $v \in H$.

(x) $\Phi:[0, T] \times H \rightarrow H$ is a mapping such that

(a) $\|\Phi(t, u)-\Phi(t, v)\|^{2} \leq \mu\|u-v\|^{2}$ for all $t \in[0, T], u, v \in H$, where $\mu$ is a positive constant;

(b) $\Phi(t, 0)=0$ for all $t \in[0, T]$;

(c) $\Phi(\cdot, v) \in \mathcal{L}_{H}^{2}[0, T]$ for all $v \in H$.

(xi) $x_{0}$ is a $H$-valued $\mathcal{F}_{0}$-measurable random variable such that $E\left\|x_{0}\right\|^{4}<\infty$.

Definition 5.1 We call a process $(U(t))_{t \in[0, T]}$ from the space $\mathcal{L}_{V}^{2}(\Omega \times[0, T])$ with $\mathbf{E}\|U(t)\|^{2}<\infty$ for all $t \in[0, T]$ a solution of the stochastic Navier-Stokes equation if it satisfies the equation:

$$
\begin{aligned}
(U(t), v)+\int_{0}^{t}\langle\mathcal{A} U(s), v\rangle \mathrm{d} s & =\left(x_{0}, v\right)+\int_{0}^{t}\langle\mathcal{B}(U(s), U(s)), v\rangle \mathrm{d} s \\
& +\int_{0}^{t}(\Phi(s, U(s)), v) \mathrm{d} s+\int_{0}^{t}(\mathcal{C}(s, U(s)), v) \mathrm{d} w(s)
\end{aligned}
$$

for all $v \in V, t \in[0, T]$ and a.e. $\omega \in \Omega$.

Remark 5.2. If we set $n=2, G \subset \mathbb{R}^{n}$ (the domain of flow),

$$
V=\left\{u \in \stackrel{\circ}{W_{2}^{1}}(G): \operatorname{div} u=0\right\}, \quad H=\bar{V}^{L^{2}(G)}
$$

and

$$
\langle\mathcal{A} u, v\rangle=\int_{G} \sum_{i=1}^{n} \frac{\partial u}{\partial x_{i}} \frac{\partial v}{\partial x_{i}} \mathrm{~d} x, \quad\langle\mathcal{B}(u, v), z\rangle=-\int_{G} \sum_{i, j=1}^{n} u_{i} \frac{\partial v_{j}}{\partial x_{i}} z_{j} \mathrm{~d} x, \quad \Phi(t, u)=f(t)
$$

for $u, v, z \in V, t \in[0, T]$, then equation (21) can be transformed into

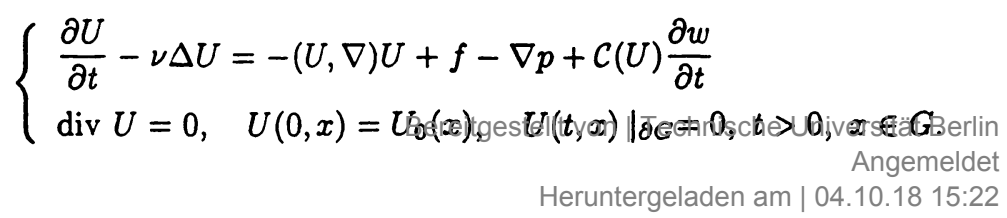


In hydromechanics this equation is known as the two dimensional stochastic NavierStokes equation.

For each $n=1,2,3, \ldots$ we consider the evolution equation with additive noise

$$
\begin{aligned}
\left(\mathbf{u}_{\mathbf{n}}(t), v\right) & +\int_{0}^{t}\left\langle\mathcal{A} \mathbf{u}_{\mathbf{n}}(s), v\right\rangle \mathrm{d} s=\left(x_{0}, v\right)+\int_{0}^{t}\left\langle\mathcal{B}\left(\mathbf{u}_{\mathrm{n}-1}(s), \mathbf{u}_{\mathbf{n}}(s)\right), v\right\rangle \mathrm{d} s \\
& +\int_{0}^{t}\left(\Phi\left(s, \mathbf{u}_{\mathrm{n}-1}(s)\right), v\right) \mathrm{d} s+\int_{0}^{t}\left(\mathcal{C}\left(s, \mathbf{u}_{\mathrm{n}-1}(s)\right), v\right) \mathrm{d} w(s),
\end{aligned}
$$

for all $v \in V, t \in[0, T]$, and a.e. $\omega \in \Omega$, where $\mathbf{u}_{0}(t)=0$ for all $t \in[0, T]$ and a.e. $\omega \in \Omega$.

Remark 5.3.

1) In equation $\left(\hat{P}_{n}\right)$, considering that $u_{n-1}$ is known, the operators $\mathcal{A}$ and $\mathcal{B}$ depend linearly on $u_{n}$ and the noise is additive with respect to $u_{n}$.

2) The approximation method given in this section holds also, if the sequence of approximations $\left(u_{n}\right)$ starts with $u_{0}(t):=x_{0}$ for all $t \in[0, T]$ and a.e. $\omega \in \Omega$.

\section{THEOREM 5.4}

(i) The Navier-Stokes equation (21) has a solution $U \in \mathcal{L}_{V}^{2}(\Omega \times[0, T])$, which is almost surely unique and has almost surely continuous trajectories in $H$.

(ii) There exists a positive constant $c$ such that

$$
\mathbf{E} \sup _{t \in[0, T]}\|U(t)\|^{4}+\mathbf{E}\left(\int_{0}^{T}\|U(s)\|_{V}^{2} \mathrm{~d} s\right)^{2} \leq c \mathbf{E}\left\|x_{0}\right\|^{4} .
$$

The proof of this result can be found in [3] (Theorem 2.2, Lemma 3.5).

THEOREM 5.5 For each $n \in \mathbb{N}$ equation $\left(\hat{P}_{n}\right)$ has an almost surely unique solution $u_{n} \in \mathcal{L}_{V}^{2} \cdot(\Omega \times[0, T])$ with almost surely continuous trajectories in $H$.

Proof. We prove the statement by induction. We apply successively Theorem 4.2 on $Z_{\Psi, \Gamma}:=u_{n}, a_{0}:=x_{0}, X:=u_{n-1}, Y:=0, a_{0}:=x_{0}, \Psi(s):=\Phi\left(s, u_{n-1}(s)\right), \Gamma:=$ $\mathcal{C}\left(s, u_{n-1}(s)\right), \mathcal{G}:=0$. The theorem is proved.

Now we establish some properties for the solutions of the equations $\left(\hat{P}_{n}\right), n \in \mathbb{N}$.

LEMMA 5.6 There exists a positive constant $c_{1}$ (depending only on $\lambda, \mu, \nu$, and $T$ ) such that each of the expressions

$$
\sup _{t \in[0, T]} \mathbf{E}\left\|u_{n}(t)\right\|^{4}, \quad \mathbf{E}\left(\int_{0}^{T}\left\|u_{n}(s)\right\|_{V}^{2} \mathrm{~d} s\right)^{2}
$$

$(n=1,2, \ldots)$ is less than or equal toBer 
Proof. Let $n \in \mathbb{N}$. We define

$$
\tilde{z}(t)=e^{-(9 \lambda+5 \sqrt{\mu}) t}, \quad t \in[0, T] .
$$

Using the Ito formula, the properties of $\mathcal{A}, \mathcal{B}, \Phi$, and $\mathcal{C}$ and some elementary calculations, we obtain

$$
\begin{aligned}
2 \nu \int_{0}^{t} \tilde{z}(s)\left\|u_{n}(s)\right\|_{V}^{2} \mathrm{~d} s \leq & \left\|x_{0}\right\|^{2}+(\lambda+\sqrt{\mu}) \int_{0}^{t} \tilde{z}(s)\left\|u_{n-1}(s)\right\|^{2} \mathrm{~d} s \\
& +2 \int_{0}^{t} \tilde{z}(s)\left(\mathcal{C}\left(s, u_{n-1}(s)\right), u_{n}(s)\right) \mathrm{d} u \cdot(s)
\end{aligned}
$$

and

$$
\begin{aligned}
& \tilde{z}(t)\left\|u_{n}(\hat{t})\right\|^{4}+4 \nu \int_{0}^{t} \tilde{z}(s)\left\|u_{n}(s)\right\|_{V}^{2}\left\|u_{n}(s)\right\|^{2} \mathrm{~d} s+2(3 \lambda+\sqrt{\mu}) \int_{0}^{t} \tilde{z}(s)\left\|u_{n}(s)\right\|^{4} \mathrm{~d} s \\
& \leq \quad\left\|x_{0}\right\|^{4}+(3 \lambda+\sqrt{\mu}) \int_{0}^{t} \tilde{z}(s)\left\|u_{n-1}(s)\right\|^{4} \mathrm{~d} s \\
& \quad+4 \int_{0}^{t} \tilde{z}(s)\left(\mathcal{C}\left(s, u_{n-1}(s)\right), u_{n}(s)\right)\left\|u_{n}(s)\right\|^{2} \mathrm{~d} w(s) .
\end{aligned}
$$

Hence, there exists a constant $c_{2}>0$ (independent of $n$ ) such that

$$
\sup _{t \in[0, T]} \mathbf{E}\left\|u_{n}(t)\right\|^{4}+2(3 \lambda+\sqrt{\mu}) \mathbf{E} \int_{0}^{T}\left\|u_{n}(s)\right\|^{4} \mathrm{~d} s \leq c_{2} \mathbf{E}\left\|x_{0}\right\|^{4} .
$$

In (23) we square both sides of the inequality. Then we use the properties of the stochastic integral and those of the Lebesgue integral to obtain

$$
\begin{aligned}
4 \nu^{2} \mathbf{E}\left(\int_{0}^{T} \tilde{z}(s)\left\|u_{n}(s)\right\|_{V}^{2} \mathrm{~d} s\right)^{2} \leq & 3 \mathbf{E}\left\|x_{0}\right\|^{4} \\
& +c_{3} \mathbf{E} \int_{0}^{T} \tilde{z}^{2}(s)\left\|u_{n-1}(s)\right\|^{4} \mathrm{~d} s+\mathbf{E} \int_{0}^{T} \tilde{z}^{2}(s)\left\|u_{n}(s)\right\|^{4} \mathrm{~d} s
\end{aligned}
$$

where $c_{3}$ is a positive constant depending on $\lambda, \mu$, and $T$. Taking into account (25) and the properties of $\tilde{z}$, it follows that there exists a constant $c_{4}$ depending on $(\lambda, \mu, \nu$ and $T$ ) such that

$$
\mathbf{E}\left(\int_{0}^{T}\left\|u_{n}(s)\right\|_{V}^{2} \mathrm{~d} s\right)^{2} \leq c_{4} \mathbf{E}\left\|x_{0}\right\|^{4} \text {. }
$$


The lemma is proved.

We define

$$
\tilde{e}(t)=\Delta_{U}(t) \exp \{-(\lambda+\sqrt{\mu}) t\}
$$

for all $t \in[0, T]$ and a.e. $\omega \in \Omega$ and introduce the following notations:

$$
\begin{aligned}
& s_{N}(t)=\sum_{n=1}^{N} \tilde{e}(t)\left\|u_{n}(t)-U(t)\right\|^{2}, \\
& S_{N}(t)=\sum_{n=1}^{N} \tilde{e}(t)\left\|u_{n}(t)-U(t)\right\|_{V}^{2},
\end{aligned}
$$

where $N$ is a natural number, $t \in[0, T], \omega \in \Omega$.

Next we will prove that the approximations $\left(u_{n}\right)$ converge in mean square to the solution $U$ of the stochastic Navier-Stokes equation (21).

THEOREM 5.7. The following convergences hold:

$$
\lim _{n \rightarrow \infty} \mathbf{E} \int_{0}^{T}\left\|u_{n}(s)-U(s)\right\|_{V}^{2} \mathrm{~d} s=0
$$

and

$$
\lim _{n \rightarrow \infty} \mathbf{E}\left\|u_{n}(t)-U(t)\right\|^{2}=0 \text { for all } t \in[0, T]
$$

Proof. Let $n \in \mathbb{N}$. Using (21), $\left(\hat{P}_{n}\right)$ and the Ito formula we obtain

$$
\begin{aligned}
& \tilde{e}(t)\left\|u_{n}(t)-U(t)\right\|^{2}+2 \int_{0}^{t} \tilde{e}(s)\left\langle\mathcal{A} u_{n}(s)-\mathcal{A} U(s), u_{n}(s)-U(s)\right\rangle \mathrm{d} s \\
& =2 \int_{0}^{t} \tilde{e}(s)\left\langle\mathcal{B}\left(u_{n-1}(s), u_{n}(s)\right)-\mathcal{B}(U(s), U(s)), u_{n}(s)-U(s)\right\rangle \mathrm{d} s \\
& -\frac{b}{\nu} \int_{0}^{t} \tilde{e}(s)\|U(s)\|_{V}^{2}\left\|u_{n}(s)-U(s)\right\|^{2} \mathrm{~d} s-(\lambda+\sqrt{\mu}) \int_{0}^{t} \tilde{e}(s)\left\|u_{n}(s)-U(s)\right\|^{2} \mathrm{~d} s \\
& +2 \int_{0}^{t} \tilde{e}(s)\left(\Phi\left(s, u_{n-1}(s)\right)-\Phi(s, U(s)), u_{n}(s)-U(s)\right) \mathrm{d} s \\
& +\int_{0}^{t} \tilde{e}(s)\left\|\mathcal{C}\left(s, u_{n-1}(s)\right)-\mathcal{C}(s, U(s))\right\|^{2} \mathrm{~d} s \\
& +2 \int_{0}^{t} \tilde{e}(s)\left(\mathcal{C}\left(s, u_{n-1}(s)\right)-\mathcal{C}(s, U(s)), u_{n}(s)-U(s)\right) \mathrm{d} w(s), \\
& \quad \text { Bereitgestellt von | Technische Universität Berlin } \\
& \text { Angemeldet }
\end{aligned}
$$


for all $t \in[0, T]$ and a.e. $\omega \in \Omega$. From the properties of $\mathcal{B}$ we can derive the following estimate:

$$
\begin{aligned}
& 2\left\langle\mathcal{B}\left(u_{n-1}(s), u_{n}(s)\right)-\mathcal{B}(U(s), U(s)), u_{n}(s)-U(s)\right\rangle \\
= & -2\left\langle\mathcal{B}\left(u_{n-1}(s)-U(s), u_{n}(s)-U(s)\right), U(s)\right\rangle \\
\leq & \frac{\nu}{2}\left\|u_{n-1}(s)-U(s)\right\|_{V}^{2}+\frac{\nu}{2}\left\|u_{n}(s)-U(s)\right\|_{i}^{2} . \\
& +\frac{b}{2 \nu}\|U(s)\|_{V}^{2}\left\|u_{n-1}(s)-U(s)\right\|^{2}+\frac{b}{2 \nu}\|U(s)\|_{V}^{2}\left\|u_{n}(s)-U(s)\right\|^{2}
\end{aligned}
$$

for all $s \in[0, T]$ and a.e. $\omega \in \Omega$. Using this estimation and the continuity of $\mathcal{C}$ in (28), we obtain

$$
\begin{aligned}
& \tilde{e}(t)\left\|u_{n}(t)-U(t)\right\|^{2}+\frac{3 \nu}{2} \int_{0}^{t} \tilde{e}(s)\left\|u_{n}(s)-U(s)\right\|_{V}^{2} \mathrm{~d} s \\
& +(\lambda+\sqrt{\mu}) \int_{0}^{t} \tilde{e}(s)\left\|u_{n}(s)-U(s)\right\|^{2} \mathrm{~d} s \\
& \leq \frac{\nu}{2} \int_{0}^{t} \tilde{e}(s)\left\|u_{n-1}(s)-U(s)\right\|_{V}^{2} \mathrm{~d} s \\
& +2 \int_{0}^{t} \tilde{e}(s)\left(\mathcal{C}\left(s, u_{n-1}(s)\right)-\mathcal{C}(s, U(s)), u_{n}(s)-U(s)\right) \mathrm{d} w(s) \\
& +\frac{b}{2 \nu} \int_{0}^{t} \tilde{e}(s)\|U(s)\|_{V}^{2}\left(\left\|u_{n-1}(s)-U(s)\right\|^{2}-\left\|u_{n}(s)-U(s)\right\|^{2}\right) \mathrm{d} s \\
& +(\lambda+\sqrt{\mu}) \int_{0}^{t} \tilde{e}(s)\left\|u_{n-1}(s)-U(s)\right\|^{2} \mathrm{~d} s
\end{aligned}
$$

fcr all $t \in[0, T]$ and a.e. $\omega \in \Omega$.

Summing up these relations for $n=1$ to an arbitrary natural number $N$, we get

$$
\begin{gathered}
s_{N}(t)+\nu \int_{0}^{t} S_{N}(s) \mathrm{d} s+\frac{b}{2 \nu} \int_{0}^{t} \tilde{e}(s)\|U(s)\|_{V}^{2}\left\|u_{N}(s)-U(s)\right\|^{2} \mathrm{~d} s \\
+(\lambda+\sqrt{\mu}) \int_{0}^{t} \tilde{e}(s)\left\|u_{N}(s)-U(s)\right\|^{2} \mathrm{~d} s \\
\leq \frac{\nu}{2} \int_{0}^{t} \tilde{e}(s)\left\|u_{0}(s)-U(s)\right\|_{V}^{2} \mathrm{~d} s+(\lambda+\sqrt{\mu}) \int_{\text {Bereitgestellt von } \mid \text { Te }}^{t} \tilde{e}(s)\left\|u_{0}(s)-U(s)\right\|^{2} \mathrm{~d} s \\
\text { Heruntergeladen Universität Berlin } \\
\text { Angemeldet }
\end{gathered}
$$




$$
\begin{aligned}
& +\frac{b}{2 \nu} \int_{0}^{t} \tilde{e}(s)\|U(s)\|_{\nu}^{2},\left\|u_{0}(s)-U(s)\right\|^{2} \mathrm{~d} s \\
& +2 \sum_{n=1}^{N} \int_{0}^{t} \tilde{e}(s)\left(\mathcal{C}\left(s, u_{n-1}(s)\right)-\mathcal{C}(s, U(s)), u_{n}(s)-U(s)\right) \mathrm{d} w(s)
\end{aligned}
$$

for all $t \in[0, T]$ and a.e. $\omega \in \Omega$, where $s_{N}$ and $S_{N}$ are defined in (26) and (2i). But $0<\tilde{e}(s) \leq 1$ and $u_{0}(s)=0$ for all $s \in[0, T]$, a.e. $\omega \in \Omega$. In the above estimation $\pi$ take the mathematical expectation, apply Theorem 5.4, then it follows that there exists a positive constant $c$, which does not depend on $N$, such that

$$
\begin{aligned}
& \mathbf{E} s_{N}(t)+\nu \mathbf{E} \int_{0}^{t} S_{N}(s) \mathrm{d} s \\
& \leq \mathbf{E} \int_{0}^{T}\left(\frac{\nu}{2}\|U(s)\|_{V}^{2}+(\lambda+\sqrt{\mu})\|U(s)\|^{2}+\frac{b}{2 \nu}\|U(s)\|_{V}^{2}\|U(s)\|^{2}\right) \mathrm{d} s \leq c
\end{aligned}
$$

for all $t \in[0, T]$ and all natural numbers $N$. Consequently, for all $t \in[0, T]$ we have

$$
\sum_{n=1}^{\infty} \mathbf{E} \tilde{e}(t)\left\|u_{n}(t)-U(t)\right\|^{2}+\nu \sum_{n=1}^{\infty} \mathbf{E} \int_{0}^{t} \tilde{e}(s)\left\|u_{n}(s)-U(s)\right\|_{V}^{2} \mathrm{~d} s \leq c
$$

Hence

$$
\lim _{n \rightarrow \infty} \mathbf{E} \int_{0}^{T} \tilde{e}(s)\left\|u_{n}(s)-U(s)\right\|_{V}^{2} \mathrm{~d} s=0
$$

and for all $t \in[0, T]$ we have

$$
\lim _{n \rightarrow \infty} \mathbf{E} \tilde{e}(t)\left\|u_{n}(t)-U(t)\right\|^{2}=0 .
$$

We take $\mathcal{T}_{M}:=\mathcal{T}_{M}^{U}$. It follows that for each fixed natural number $M$ we have

$$
\lim _{n \rightarrow \infty} \mathbf{E} \int_{0}^{\mathcal{T}_{M \prime}}\left\|u_{n}(s)-U(s)\right\|_{V^{\prime}}^{2} \mathrm{~d} s=0 \text { and } \lim _{n \rightarrow \infty} \mathbf{E}\left\|u_{n}\left(\mathcal{T}_{M}\right)-U\left(\mathcal{T}_{M}\right)\right\|^{2}=0
$$

First we apply Proposition 4.1 for $\mathcal{T}:=T$,

$$
Q_{n}(\mathcal{T}):=\int_{0}^{\tau}\left\|u_{n}(s)-U(s)\right\|^{2} \mathrm{~d} s
$$




$$
\lim _{n \rightarrow \infty} \mathbf{E} \int_{0}^{T}\left\|u_{n}(s)-U(s)\right\|_{V}^{2} \mathrm{~d} s=0 .
$$

Let $t \in[0, T]$. Now we apply Proposition 4.1 for $\mathcal{T}:=t, Q_{n}(\mathcal{T}):=\left\|u_{n}(\mathcal{T})-U(\mathcal{T})\right\|$, use Lemma 5.6 and Theorem 5.4 to get

$$
\lim _{n \rightarrow \infty} \mathbf{E}\left\|u_{n}(t)-U(t)\right\|^{2}=0 .
$$

Thus Theorem 5.7 is proved.

\section{REFERENCES}

1. A. Bensoussan. A Model of Stochastic Differential Equation in Hilbert Spaces Applicable to Navier-Stokes Equation in Dimension 2. Stochastic analysis, Proc. Honor Moshe Zakai 65th Birthday, Haifa, Israel 1991, 51-73 (1991).

2. H. Breckner (Lisei), Approximation and Optimal Control of the Stochastic NavierStokes Equation. Dissertation, Martin-Luther University, Halle-Wittenberg, Germany (1999).

3. H. Breckner (Lisei). Galerkin Approximation and the Strong Solution of the Stochastic Navier-Stokes Equation, Jornal of Applied Mathematics and Statistic Analysis 13, 3, 239-259 (2000).

4. M. Capinski, D. Gatarek. Stochastic equations in Hilbert space with application to Navier-Stokes equations in any dimension, J. Funct. Anal. 126, 1, 26-35 (1994).

5. J. F. Clouet. A Diffusion-Approximation Theorem in Navier-Stokes Equation, Stochastic Anal. Appl. 14, 1, 33-46 (1996).

6. F. Flandoli, V. M. Tortorelli, Time discretization of Ornstein-Uhlenbeck equations and stochastic Navier-Stokes equations with a generalized noise, Stochastics and Stochastics Reports 55, 1-2, 141-165 (1995).

7. W. Grecksch, B. Schmalfuß, Approximation of the stochastic Navier-Stokes equation. Comp. Appl. Math. V. 15, no. 3, 227-239 (1997).

8. P. E. Kloeden, E. Platen. Numerical Solution of Stochastic Differential Equations. Springer Verlag, New York - Berlin (1995).

9. A.I. Komech, M.I. Vishik. Statistical solutions of the Navier-Stokes and Euler equations. Advances in Mechanics 5, no. 1-2, 65-120 (1982) (in Russian).

10. J.L. Lions. Optimal Control of Systems Governed by Partial Differential Equations. Springer Verlag, Berlin - New York (1971).

11. H. Lisei. Existence of Optimal and $\varepsilon$-Optimal Controls for the Stochastic NavierStokes Equation (submitted for publication to Nonlinear Analysis, 2000).

12. B. Oksendal. Stochastic Differential Equations. Springer Verlag, Berlin - New York (1985).

13. B.L. Rozovskij. Stochastic Evolution Systems. Kluwer Academic Publishers, Dordrecht (1990).

14. B. Schmalfuß, Bemerkungen zur zweidimensionalen stochastischen Navier-StokesGleichung, Math. Nachr. 131, 19-32 (1987). 
15. B. Schmalfuß. Endlichdimensionale Approximation der stochastischen NavierStokes-Gleichung, Statistics 2, 149-157 (1990).

16. K. Twardowska. An Approximation Theorem of Wong-Zakai type for Stochastic Navier-Stokes Equations. Rend. Semin. Mat. Univ. Padova 96, 15-36 (1996).

17. M. Viot. Solution faible d'équation aux dérivés partielles stochastiques non linéaires, Thése doct. sci. math., Paris (1976).

18. M.I. Vishik, A.V. Fursikor. Mathematical Problems of Statistical Hydromechanics. Kluwer Academic Publishers, London (1980).

19. E. Zeidler, Nonlinear Functional Analysis and its Applications. Vol. I: Fixed-Point Theorems. Springer Verlag: New York - Berlin (1986).

20. E. Zeidler, Nonlinear Functional Analysis and its Applications. Vol. II/A, II/B: Linear Monotone Operators. Springer Verlag, New York - Berlin (1990). 